travel restrictions. Questions surveyed stakeholder opinions of prior assessed emergency health needs and their ability to address these shortcomings in the following year. Open-ended questions queried for critical problems facing emergency health.

The survey was completed by 31 citizens of Jerusalem and the West Bank and 17 from Gaza. None of the physicians had emergency training, $40 \%$ had general nursing or medical training, $17 \%$ had emergency nursing training, and $35 \%$ had trained in another medical specialty. Most nurses had practiced for 15 years and physicians from Gaza had more experience than their counterparts (average 22 years versus 11 years, respectively). All respondents felt both triage and improved charting was needed, yet only $58 \%$ felt changes could be implemented. Triage was identified as the role of senior nurses by $58 \%$, and a shared medical doctor/registered nurse role by $44 \%$. ICD-10 coding was supported by $88 \%$, yet only $40 \%$ felt it could be implemented. Improved continuing education was supported by $98 \%$, graduate emergency education had $100 \%$ support, ED quality improvement was supported by $98 \%$, and injury surveillance was supported by $92 \%$. Leading problems in emergency care included ED security, staff shortages, a lack of facilities, a lack of continuing education, a lack of emergency specialists, overcrowding, a lack of qualified staff, a need for public education, a lack of triage, and a lack of standards.

Considerable challenges impede Palestinian emergency care. It is clear that providers are aware of the key foundations of emergency care, yet are doubtful improvements can be implemented in the current uprising. Continued professional development activities with the support of the international community are necessary.

Keywords: assessment; challenges; emergency department; emergency health; Gaza Strip; Jerusalem; West Bank Prehosp Disast Med 2005;20(2):s51-s52

\section{The Consequences of an Earthquake in Vrancea on Population and the Healthcare System \\ N. Steiner; ${ }^{1}$ D. Ion ${ }^{2}$ \\ 1. Romanian National Centre For Training in Medical Management of Disasters, Romania \\ 2. Romanian Health Ministry, Romania}

Objective: To continue the previous evaluations of morbidity and specific mortality due to an earthquake, measuring a magnitude of 7.2 on the Richter scale, and having the main seismic wave a duration of 10-15 seconds.

Methods: Taking into account the above mentioned data, an earthquake with the main focus in the Vrancea area, a magnitude of 8 on the Richter scale, and the duration of the main wave lasting 15 seconds was simulated.

Results: The computer program Epi Info 6.04D was used and a unique calculation algorithm was developed to obtain the results shown in the presentation. The main results of the simulation were: (1) 22,147 total expected casualties; (2) 3,336 total deceased; and (3) 4,034 total trapped casualties. Conclusions: It was noted that an evaluation of urban vulnerability, an estimation of the expected number of casual- ties, and an evaluation of hospital and prehospital system's capacity and structures are needed. Also, an accurate prehospital intervention system and a medical emergency system as the infrastructure for the medical intervention system in disaster situations must be developed. It was found that proper endowment and preparedness of the entire medical intervention system is needed, and a firm leadership in the medical intervention system and communication system must be provided.

Keywords: disaster; earthquake; preparedness

Prebosp Disast Med 2005;20(2):552

The Cafe Fire on New Year's Eve in Volendam, Netherlands: Description of Events

\section{Welling, ${ }^{1}$ S.M. van Harten; ${ }^{2}$ P. Patka, ${ }^{2}$}

J.J.L.M. Bierens, ${ }^{2}$ M. Boers, ${ }^{2}$ J.S.K. Luitse, ${ }^{1}$ D.P. Mackie, ${ }^{3}$

A. Trouwborst ${ }^{1}$ D.J. Gouma, ${ }^{1}$ R.W. Kreis ${ }^{3}$

1. Academic Medical Center, Netherlands

2. VU Medical Center, Netherlands

3. Red Cross Hospital, Beverwijk, Netherlands

Introduction: The cafe fire at Volendam occurred shortly after midnight on 01 January 2001, and resulted in one of the worst mass burn incidents in recent Dutch history. The aim of this study was to provide insight into the medical and organizational requirements of a major burn incident. Objectives: Shortly after the fire, two university hospitals and a burn center in the region of the accident developed an initiative to evaluate the medical care given during and after this major burns incident. A multidisciplinary research group investigated the medical management of the victims at the scene, in the emergency departments (EDs) and during admission in the hospitals. All 245 casualties were included in this study.

Methods: A fire occurred in a crowded cafe with approximately 350 young visitors on a small embankment of a relatively isolated town, resulting in an unusually high number of severely injured burn victims.

Results: Four died immediately. The ensuing rescue effort was hampered by poor access and chaotic circumstances. At the scene of the event, mobile medical teams ensured an order of transport or treatment priority of the injured. There were 245 victims with a median total body surface area (TBSA) burned of $12 \%$. Inhalation injury was present in 96 patients. A total of 182 victims had to be admitted, of whom 112 were admitted to Intensive Care. Ten patients died in the hospital. Seventy-eight patients were secondarily transported, many to specialized centers in the Netherlands and abroad. In total, 36 hospitals in three countries participated in the care.

Conclusion: An accident with a high number of burn victims imposes a challenge on health care. The difficult circumstances at the site demonstrated the need for robust organizational structures. The primary and secondary dispersal of patients required strong coordination and general hospitals were able to provide initial medical care to major burn casualties.

Keywords: burn; fire; Netherlands

Prebosp Disast Med 2005;20(2):552 